\title{
SURFACES OF REVOLUTION IN THE THEORY OF LAME'S PRODUCTS.
}

BY PROFESSOR F. H. SAFFORD.

(Read before the American Mathematical Society September 4, 1916.)

IN Berliner Monatsberichte, February, 1878, Wangerin discussed the problem of the most general orthogonal surfaces of revolution such that if Laplace's equation be written in coordinates corresponding to these surfaces a solution may be obtained in the form of a Lamés product with an extraneous factor, i. e.,

$$
V=\lambda \cdot R_{1} \cdot R_{2} \cdot \theta .
$$

$R_{1}, R_{2}$, and $\theta$ are functions respectively of the parameters of the two families of surfaces and the meridian planes, while $\lambda$ may involve all three parameters. Wangerin showed that $\lambda$ is $1 / \sqrt{ } r$, where $r$ is the distance from the axis of revolution to the point of intersection of the three surfaces. He also deduced certain meridian curves which have been discussed by Haentzschel in Reduction der Potentialgleichung, Berlin, 1893.

In reducing Laplace's equation the terms involving $\theta$ are removed in the usual manner, leaving a partial differential equation which, as both writers state, is resolvable into two ordinary differential equations provided $F$ and $F_{1}$, its conjugate, can be found which shall satisfy

$$
\frac{F^{\prime}(t+i u) \cdot F_{1}^{\prime}(t-i u)}{\left[F(t+i u)-F_{1}(t-i u)\right]^{2}}=H(t)+K(u) .
$$

After $F$ is found the two families of meridian curves follow from

(3) $x+i r=F(t+i u), x-i r=F_{1}(t-i u), r=\sqrt{ }\left(y^{2}+z^{2}\right)$,

by elimination of $u$ and $t$ respectively, thus making $t$ and $u$ the parameters of which $R_{1}$ and $R_{2}$ are functions as stated above. As will be seen, $H$ and $K$ disappear in the process of finding $F$, and the principal result in this paper is a direct process of computing $H$ and $K$, avoiding the method of 
substitution in the first member of (2), which must be followed by a separation not always obvious.

Wangerin differentiates (2) successively with respect to $t$ and $u$ respectively, obtaining

$$
\begin{aligned}
& \left(F-F_{1}\right)\left[\left(F-F_{1}\right)\left(F^{\prime \prime \prime} F_{1}{ }^{\prime}-F^{\prime} F_{1}{ }^{\prime \prime}\right)\right. \\
& \quad+\left(F^{\prime \prime} F_{1}{ }^{\prime}-F^{\prime} F_{1}{ }^{\prime \prime}\right)\left(F^{\prime}-F_{1}{ }^{\prime}\right) \\
& \left.\quad-2 F^{\prime} F_{1}{ }^{\prime}\left(F^{\prime \prime}+F_{1}^{\prime \prime}\right)-2\left(F^{\prime}+F_{1}{ }^{\prime}\right)\left(F^{\prime} F_{1}{ }^{\prime \prime}+F^{\prime \prime} F_{1}{ }^{\prime}\right)\right] \\
& \quad-3\left(F^{\prime}-F_{1}\right)\left[\left(F-F_{1}\right)\left(F^{\prime \prime} F_{1}{ }^{\prime}-F^{\prime} F_{1}{ }^{\prime \prime}\right)\right. \\
& \left.\quad-2 F^{\prime} F_{1}{ }^{\prime}\left(F^{\prime}+F_{1}\right)\right]=0 .
\end{aligned}
$$

This expression is next differentiated three times with respect to the argument of $F$ and then the derivatives of $F_{1}$ are eliminated. From this result comes the final quotation from Wangerin, the following equation defining $F$ :

$$
\text { “ }\left(F^{\prime}\right)^{2}=A F^{4}+4 B F^{3}+6 C F^{2}+4 B^{\prime} F+A^{\prime}=R(F) . "
$$

Corresponding to (5)

$$
\left(F_{1}{ }^{\prime}\right)^{2}=A_{1} F_{1}{ }^{4}+4 B_{1} F_{1}{ }^{3}+6 C_{1} F_{1}{ }^{2}+4 B_{1}{ }^{\prime} F_{1}{ }^{\prime}+A_{1}{ }^{\prime} .
$$

The conjugate imaginary constants in (5) and (6) come from integration but must be taken real, because (4) is satisfied by by $F, F_{1}$ and their derivatives from (5) and (6) when and only when the following conditions hold:

(7) $A-A_{1}=B-B_{1}=C-C_{1}=B^{\prime}-B_{1}{ }^{\prime}=A^{\prime}-A_{1}{ }^{\prime}=0$.

Haentzschel gives as the solution of (5)

(8) $\quad F(z)=z_{0}$

$$
\begin{aligned}
& +\frac{\sqrt{ } R\left(z_{0}\right) \sqrt{ } S+\frac{1}{2} R^{\prime}\left(z_{0}\right)\left[s-\frac{1}{24} R^{\prime \prime}\left(z_{0}\right)\right]+\frac{1}{24} R\left(z_{0}\right) R^{\prime \prime \prime}\left(z_{0}\right)}{2\left[s-\frac{1}{24} R^{\prime \prime}\left(z_{0}\right)\right]^{2}-A \cdot R\left(z_{0}\right)}, \\
& S=4 s^{3}-g_{2} s-g_{3}=4\left(s-\epsilon_{1}\right)\left(s-\epsilon_{2}\right)\left(s-\epsilon_{3}\right), \\
& s=\wp(z), \quad g_{2}=A A^{\prime}-4 B B^{\prime}+3 C^{2}, \\
& \quad g_{3}=A C A^{\prime}+2 B C B^{\prime}-A B^{\prime 2}-A^{\prime} B^{2}-C^{3} .
\end{aligned}
$$

The constant $z_{0}$ is in general unrestricted. When $z_{0}$ is a root 
of $R(z)=0,(8)$ reduces to

$$
F(z)=z_{0}+\frac{\frac{1}{4} R^{\prime}\left(z_{0}\right)}{s-\frac{1}{24} R^{\prime \prime}\left(z_{0}\right)} .
$$

Formula (8) was published in 1865 in a pamphlet entitled "Problemata quaedam mechanica functionum ellipticarum ope soluta.-Dissertatio inauguralis," by G. G. A. Biermann (Berolini), where it is quoted as derived from Weierstrass's lectures.

For $F(t+i u)$ given by (10) and also for

$$
F(t+i u)=\wp(t+i u),
$$

which is obtainable from (10) by linear transformation, Haentzschel has found (l. c.) the values of $H$ and $K$ in (2) by substitution of the respective values of $F$ and $F_{1}$ in the first member. This method evidently requires a considerable knowledge of the properties of $F$ and $F_{1}$ in order that the separation of the first member into two functions may be effected, thus obtaining the values of $H$ and $K$ as desired, and an inspection of Haentzschel's presentation will confirm this inference.

This paper gives a method of expressing $H$ and $K$ as functions of $F$ and $F_{1}$ and requires only known properties of $\wp^{(z)}$ in addition to the differential equation (5), thus giving explicitly the separation implied in (2). From (2) by differentiation as to $t$

$$
\text { (11) } \frac{\left(F-F_{1}\right)\left(F^{\prime} F_{1}{ }^{\prime \prime}+F_{1}{ }^{\prime} F^{\prime \prime}\right)-2 F^{\prime} F_{1}{ }^{\prime}\left(F^{\prime}-F_{1}{ }^{\prime}\right)}{\left(F-F_{1}\right)^{3}}=H^{\prime}(t) \text {, }
$$

in which the arguments of $F$ and $F_{1}$ are respectively $t+i u$ and $t-i u$, and it should be noted that $F$ and $F_{1}$ are known functions previously determined by (5) and (6) and given by (8).

Although (11) appears to contain functions of $u$ in the first member, the second member shows that it is independent of $u$ and thus true for any value of $u$, e. g., $(t-c) / i$. This value of $u$ was suggested by the fact that $F_{1}(t-i u)$ thus becomes $F_{1}(c)$. If next $c$ is so chosen as to satisfy

$$
F_{1}{ }^{\prime}(c)=0,
$$

equation (11) will be much simplified. Since $F$ given by (8) 
is a function of $\wp,(12)$ is satisfied by taking $c$ as $\omega$, the half period of $\wp$, noticing that $\wp^{\prime}(\omega)$ is 0 . Hence (11) becomes

$$
\frac{F_{1}^{\prime \prime}(\omega) F^{\prime}(2 t-\omega)}{\left[F(2 t-\omega)-F_{1}(\omega)\right]^{2}}=H^{\prime}(t) .
$$

Integrating with respect to $t$ gives

$$
H(t)=\frac{-F_{1}^{\prime \prime}(\omega)}{2\left[F(2 t-\omega)-F_{1}(\omega)\right]}+C_{1} .
$$

Similarly

$$
K(u)=\frac{F_{1}^{\prime \prime}(\omega)}{2\left[F(2 i u+\omega)-F_{1}(\omega)\right]}+C_{2} .
$$

The final form of (2) is

$$
\begin{aligned}
& \frac{F^{\prime}(t+i u) \cdot F_{1}{ }^{\prime}(t-i u)}{\left[F(t+i u)-F_{1}(t-i u)\right]^{2}}=H(t)+K(u) \\
& =\frac{F_{1}^{\prime \prime}(\omega)}{2}\left[\frac{1}{F(2 i u+\omega)-F_{1}(\omega)}-\frac{1}{F(2 t-\omega)-F_{1}(\omega)}\right] .
\end{aligned}
$$

The term $C_{1}+C_{2}$ from the previous equations is omitted as (16) is an identity for $i u=t-\omega$.

Before treating the general value of $F$ by the use of (16) several special cases will be computed. For the simplest case, $F=\wp$, obtainable from (10) by a linear transformation with real coefficients, the following are necessary in applying (16):

$$
\begin{aligned}
& \wp(\omega)=\epsilon_{1}, \wp^{\prime}(\omega)=0, \wp^{\prime \prime}(\omega)=2\left(\epsilon_{2}-\epsilon_{1}\right)\left(\epsilon_{3}-\epsilon_{1}\right), \\
& \wp(2 t-\omega)-\epsilon_{1}=\frac{\left(\epsilon_{2}-\epsilon_{1}\right)\left(\epsilon_{3}-\epsilon_{1}\right)}{\wp(2 t)-\epsilon_{1}}, \\
& \wp(2 i u+\omega)-\epsilon_{1}=\frac{\left(\epsilon_{2}-\epsilon_{1}\right)\left(\epsilon_{3}-\epsilon_{1}\right)}{\wp(2 i u)-\epsilon_{1}},
\end{aligned}
$$

whence

$$
H(t)+K(u)=\wp(2 i u)-\wp(2 t) .
$$

When $F$ is given by (10) and $z_{0}$ is a real root of $R(z)=0$, the result is (18) as in the preceding case, the computation being somewhat longer, though depending only on (17). In this case the coefficients in (10) are real, so that either of the values 
of $F$ in the two cases is a real linear transformation of the other. It is a remarkable fact that under such a transformation the fundamental formula (16) is invariant, this being true also of the more general form which (16) would have if $c$ were not specialized.

To verify the proposition above for the last member of (16), let $F(2 t-\omega)$ be replaced by $(\alpha F+\beta) /(\gamma F+\delta), \alpha, \beta, \gamma, \delta$ real, and $\alpha \delta-\beta \gamma=\Delta \neq 0$, and similarly for $F(2 i u+\omega)$, writing also a like function of $W$ in place of $F_{1}(\omega)$, noticing that $W^{\prime}$ will be zero. The result is

$$
\begin{array}{r}
\frac{W^{\prime \prime} \Delta}{2(\gamma W+\delta)^{2}}\left[\frac{\left(\gamma F_{1}+\delta\right)(\gamma W+\delta)}{\left(F_{1}-W\right) \Delta}-\frac{(\gamma F+\delta)(\gamma W+\delta)}{(F-W) \Delta}\right] \\
=\frac{W^{\prime \prime}}{2}\left[\frac{1}{F_{1}-W}-\frac{1}{F-W}\right]
\end{array}
$$

When at least two of the roots of $R(z)=0$ are imaginary let $z_{0}$ be one of the conjugate pair $a_{1}$ and $a_{4}$; then from (10)

$$
\begin{aligned}
F(t+i u) & =a_{1}+\frac{\frac{1}{4} R^{\prime}\left(a_{1}\right)}{\wp(t-i u)-\frac{R^{\prime \prime}\left(a_{1}\right)}{24}} \\
& =a_{1}+\frac{M_{1}}{\wp(t+i u)-N_{1}}, \\
F_{1}(t-i u) & =a_{4}+\frac{\frac{1}{4} R^{\prime}\left(a_{4}\right)}{\wp(t-i u)-\frac{R^{\prime \prime}\left(a_{4}\right)}{24}} \\
& =a_{4}+\frac{M_{4}}{\wp(t-i u)-N_{4}}, \\
F_{1}(\omega) & =a_{4}+M_{4} /\left(\epsilon_{1}-N_{4}\right), F_{1}{ }^{\prime}(\omega)=0, \\
F_{1}^{\prime \prime}(\omega) & =-M_{4} \wp^{\prime \prime}(\omega) /\left(\epsilon_{1}-N_{4}\right)^{2} .
\end{aligned}
$$

These values substituted in (16) give

(21) $H(t)+K(u)=$

$$
\frac{M_{4}^{(\wp \prime \prime}(\omega)}{2\left(\epsilon_{1}-N_{4}\right)^{2}}\left[\frac{1}{a_{1}-a_{4}+\frac{M_{1}}{\wp(2 t-\omega)-N_{1}}-\frac{M_{4}}{\epsilon_{1}-N_{4}}}\right.
$$




$$
\left.-\frac{1}{a_{1}-a_{4}+\frac{M_{1}}{\wp(2 i u+\omega)-N_{1}}-\frac{M_{4}}{\epsilon_{1}-N_{4}}}\right] .
$$

For the reduction of (21) the following formulas are useful:

$$
\begin{gathered}
\begin{array}{c}
R(z)=A\left(z-a_{1}\right)\left(z-a_{2}\right)\left(z-a_{3}\right)\left(z-a_{4}\right), \\
R^{\prime}\left(a_{1}\right)=A\left(a_{1}-a_{2}\right)\left(a_{1}-a_{3}\right)\left(a_{1}-a_{4}\right)=4 M_{1}, \\
R^{\prime \prime}\left(a_{1}\right)=2 A\left[\left(a_{1}-a_{2}\right)\left(a_{1}-a_{3}\right)+\left(a_{1}-a_{3}\right)\left(a_{1}-a_{4}\right)\right. \\
\left.\quad+\left(a_{1}-a_{4}\right)\left(a_{1}-a_{2}\right)\right]=24 N_{1}
\end{array} \\
\quad\left[\text { similarly for } M_{4} \text { and } N_{4}\right], \\
\epsilon_{1}=\frac{A}{12}\left[\left(a_{1}-a_{2}\right)\left(a_{3}-a_{4}\right)+\left(a_{1}-a_{3}\right)\left(a_{2}-a_{4}\right)\right], \\
\epsilon_{2}=\frac{A}{12}\left[\left(a_{2}-a_{3}\right)\left(a_{1}-a_{4}\right)+\left(a_{2}-a_{1}\right)\left(a_{3}-a_{4}\right)\right], \\
\epsilon_{3}=\frac{A}{12}\left[\left(a_{3}-a_{1}\right)\left(a_{2}-a_{4}\right)+\left(a_{3}-a_{2}\right)\left(a_{1}-a_{4}\right)\right], \\
\epsilon_{1}-N_{4}=\frac{A}{4}\left(a_{3}-a_{4}\right)\left(a_{4}-a_{2}\right), \\
\epsilon_{1}-N_{1}=\frac{A}{4}\left(a_{1}-a_{2}\right)\left(a_{3}-a_{1}\right), \\
a_{1}-a_{4}-\frac{M_{4}}{\epsilon_{1}-N_{4}}=0 .
\end{gathered}
$$

With the aid of (17) also, the result for this case is

$$
\text { (23) } \begin{aligned}
H(t)+K(u)=-\left(\epsilon_{1}-\epsilon_{2}\right)\left(\epsilon_{1}-\epsilon_{3}\right) & {\left[\frac{1}{\wp(2 t)-\epsilon_{1}}\right.} \\
& \left.-\frac{1}{\wp(2 i u)-\epsilon_{1}}\right] .
\end{aligned}
$$

It will be noticed that $\epsilon_{2}$ and $\epsilon_{3}$, which are conjugate, enter symmetrically, $\epsilon_{1}$ being real. When all of the roots of $R(z)=0$ are imaginary and accordingly all of the $\epsilon$ 's are real, the choice of $z_{0}$ as in the preceding case reproduces (23). 
The final forms for each case up to this point are the same as given by Haentzschel, though the method is different, in that one formula, (16), gives the resolved form for all cases.

For the last and most general case, where $F$ is given by (8), the complete resolution into separate functions of $t$ and $u$ respectively would otherwise be obtainable only with much difficulty.

Although (16), the principal formula in this paper, is complete theoretically, there are three ways in which simplifications may be made in the general case. The first depends upon changes in the form of $F$ which the writer has given in the Archiv der Mathematik und Physik, III, volume 23, Heft 1, and volume 24 , Heft 4 . The second involves certain simplifications in $F_{1}(\omega)$ which, with other related matters, are intended for a later paper, while the third consists of the following substitutions derived from the present discussion. In the last member of (16), $s$ in $F$ in the second denominator is replaced by $\wp(2 t-\omega)$, also

$$
S=\frac{4\left(\epsilon_{1}-\epsilon_{2}\right)^{2}\left(\epsilon_{1}-\epsilon_{3}\right)^{2}\left[{ }^{\wp}(2 t)-\epsilon_{2}\right]\left[{ }^{\wp}(2 t)-\epsilon_{3}\right]}{\left[\curlyvee(2 t)-\epsilon_{1}\right]^{3}}
$$

and similarly for the first denominator.

In forming $F_{1}(\omega), z_{0}$ is replaced by its conjugate, but $s$ is simply $\epsilon_{1}$ which is real, while $S$ is zero. i. e.,

$F_{1}^{\prime \prime}(\omega)$ may be computed from (6) thus depending on $F_{1}(\omega)$,

$$
F_{1}{ }^{\prime \prime}(\omega)=2\left[A F_{1}{ }^{3}(\omega)+3 B F_{1}{ }^{2}(\omega)+3 C F_{1}(\omega)+B^{\prime}\right] .
$$

As a summary, it may be stated that Wangerin made the solution of the original potential problem depend upon expressing a certain function of an unknown function as a sum of two functions $H(t)$ and $K(u)$, and obtained some simple values of the unknown function. Haentzschel applied a formula due to Weierstrass thus finding a general value of the unknown function, and he also computed the values of the two functions $H$ and $K$ for special cases only. Formulas (16) and (25) above give $H$ and $K$ explicitly in terms of the general function mentioned.

University of Pennsylvania, August 1, 1916. 